\title{
Remerciements à nos évaluateurs de 2019
}

Nous tenons à remercier les personnes suivantes pour leur contribution inestimable en tant qu'évaluateurs pour la revue Promotion de la santé et prévention des maladies chroniques au Canada en 2019. Leur expertise contribue grandement à la qualité de notre revue et à la diffusion des nouvelles connaissances auprès de la communauté scientifique, au Canada comme à l'échelle internationale.

Peg Allen

Brittany Barker

Maulik Baxi

Kathy L. Belton

Nicholas Birkett

Sarah Bowen

Randall Boyes

Annie Bronsard

Alexandra Butler

Russ Callaghan

Elisabetta Camussi

Sarah Carsley

Neil E. Collishaw

Jermaine Dambi

Isabelle Doré

Christopher Ellison

Peter Gates

Amélie Gauthier-Beaupré

Mélissa Généreux

Naomi Gibbs

Gary Goldfield

Maritia Gully

Brian D. Gushulak

Naomi Hamm

Gregory W. Heath

Billie Jane Hermosura

Jeff Housman
Patricia Huston
Geoff Hynes
Pamela Kaufman
Soyeon Kim
Nathan King
Hans Krueger
Yvonne Laird
Stephanie Lake
Steven Lam
Allana LeBlanc
Andrée-Anne Ledoux
Mario Lintz
Emily Lowthian
Marjorie MacDonald
Alison Macpherson
Molly Sweeney Magee
Gina Martin
André McDonald
Jennifer Ann McGetrick
Leia Minaker
Olivia Moir
Ryan Ng
Stephanie Nishi
Jon Noel
Patrick Norman
Mation

Leon Nshimyumukiza

Hee Young Paik

William Pickett

Thomas W. Piggott

Abbey Poirier

Jason D. Pole

Jane Y. Polsky

Hudson Reddon

Justine Renard

Yibing Ruan

Susan Rudy

Robert Schwartz

Nicole Shaver

Adam Sherk

Richard Stanwick

Anne Mari Steigen

Tamara Thompson

Kate Vallance

Jenna van Draanen

Lydi-Anne Vézina-Im

Jeannette Waegemakers Schiff

Ashley Wettlaufer

Elizabeth Wright

Rongxiu Wu 